\title{
Rapid Response Missions to Explore Fast, High-Value Targets such as Interstellar Objects and Long Period Comets
}
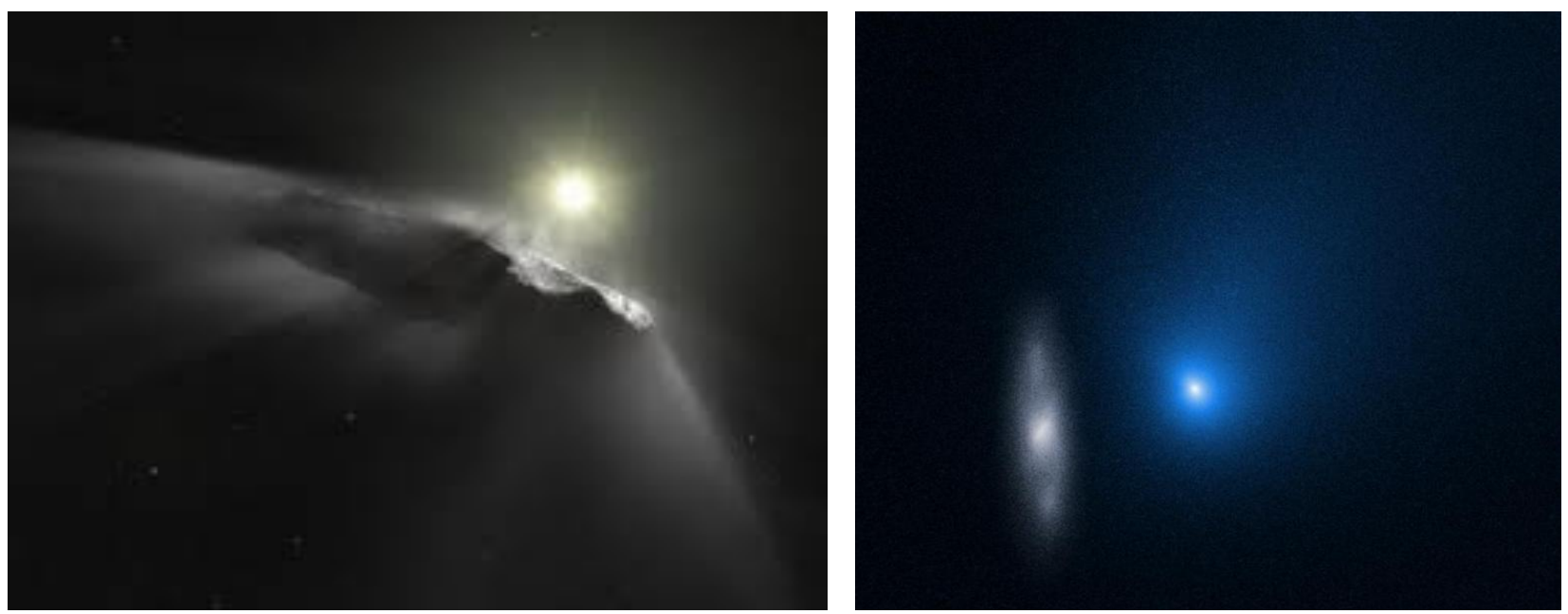

\section{Lead Authors:}

Kimberly M. Moore (California Institute of Technology), kmmoore@caltech.edu Julie Castillo-Rogez (Jet Propulsion Laboratory, California Institute of Technology) Karen J. Meech (University of Hawai'i, Institute for Astronomy) Samuel W. Courville (Planetary Science Institute, Arizona State University) Benjamin P.S. Donitz (Jet Propulsion Laboratory, California Institute of Technology) Sierra Ferguson (Arizona State University)

Kristie Llera (Southwest Research Institute, San Antonio)

Richard French (Rocket Lab USA, Inc.)

\section{Endorsers:}

Ellen C. Czaplinski (U. of Arkansas), Donald H. Kuettel III (CU Boulder), Hope Ishii (U. Hawaii), Jan Kleyna (U. Hawaii), Erica Molnar-Bufanda (U. of Hawaii), Rachana Agrawal (Purdue), Ashley Schoenfeld (UCLA), Karl Mitchell (JPL/Caltech), Peter Buhler (Planetary Science Institute), Damon Landau (JPL/Caltech), Stefano Nerozzi (Lunar \& Planetary Laboratory, U. of Arizona), Joseph G. O'Rourke (Arizona State U.), Ariel N. Deutsch (Brown), Jeffrey Stuart (JPL/Caltech), Richard Wainscoat (U. Hawaii), Richard Archer (NASA ARES), Jesse Tarnas (Brown), Steve Matousek (JPL/Caltech), Tibor Balint (JPL/Caltech)

\section{Acknowledgements \& Disclaimer:}

Part of this research was carried out at the Jet Propulsion Laboratory, California Institute of Technology, under a contract with the National Aeronautics and Space Administration. Pre-Decisional Information - For Planning and Discussion Purposes Only. The cost information contained in this document is of a budgetary and planning nature and is intended for informational purposes only. It does not constitute a commitment on the part of JPL and/or Caltech. 


\section{Executive Summary}

We advocate for enabling rapid response strategies to reach fast, high-value science targets such as interstellar objects (ISOs), Oort Cloud Comets (OCCs), and Planetary Defense targets (Near Earth Objects, NEOs). Traditional NASA Planetary Science missions require a specific target to be named upon proposal submission, with wait times of up to 6-10 years between their initial proposal and final launch (e.g. NASA Discovery 2019 AO). In contrast, "rapid response" is a flexible strategy where a spacecraft is launched in response to the discovery of a compelling target. Rapid response strategies are required for fast targets since these objects typically have short windows of discovery before their closest approach to Earth. We advocate for specific revisions to NASA policies to enable rapid response architectures including flexible targets, launch dates, pre-planned spacecraft storage, and an expansion of the SmallSat program. Although this white paper focuses on missions to Oort Cloud Comets and interstellar objects, the policy recommendations we present here would also enable missions to a broader range of targets.

\section{Interstellar and Oort Cloud Comet Science}

OCCs and ISOs are compelling targets that address the next decade's important priority science questions. OCCs and ISOs are believed to be remnants of giant planet migration, which have been scattered to the Oort Cloud or ejected out of their solar system entirely. Since OCCs and ISOs record their chemical and thermal formation conditions, they can provide clues to the earliest epochs of solar system evolution, both in our system and exoplanetary systems (Raymond and Izidoro, 2017). Telescopic capabilities are now sufficiently advanced to regularly discover these objects (as evidenced by the detections of interstellar objects 1I/2017 'Oumuamua and 2I/2019 Borisov, and OCC C/2017 K2), but investigating their physical and chemical properties require dedicated spacecraft missions.
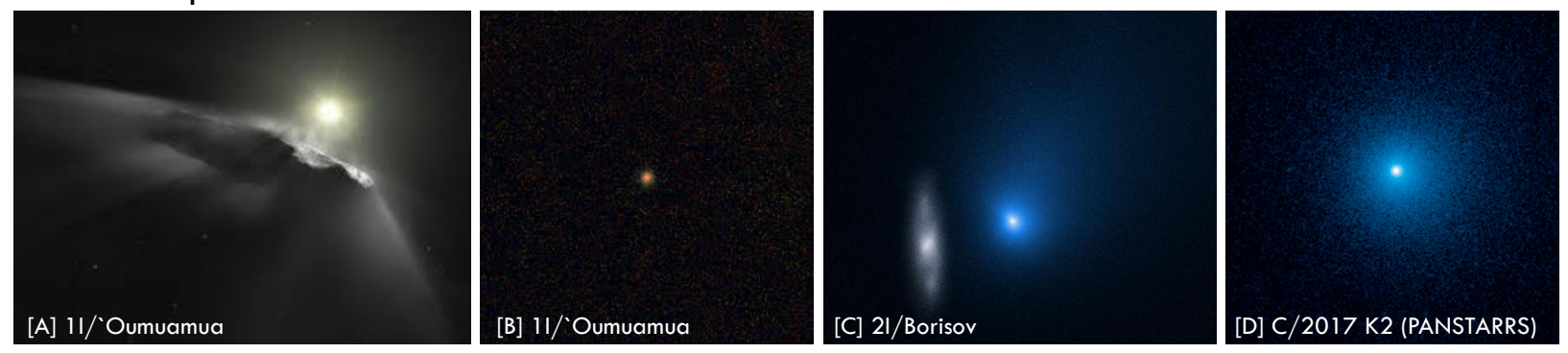

Figure 1: [A] Artistâs representation of 1I/âOumuamua European Southern Observatory/M. Kornmesser). [B] 1I/'Oumuamua seen from Gemini North telescope, scaled for true surface reflectivity (Gemini Observatory/AURA/NSF), [C] ISO 2I/Borisov from the Hubble Space Telescope (NASA/ESA/Hubble), [D] LPC C/2017 K2 (NASA/ESA/Hubble).

Oort cloud comets (OCCs) are objects that originated in the Oort Cloud, which is located roughly between $\sim 2,000-200,000$ AU (Dones et al., 2015). Galactic tidal forces can perturb these objects into long period orbits ( $>200$ yrs; some have periods $>1$ million yrs) (Dones et al., 2015). Due to their long orbits, any volatiles present may remain relatively unmodified (Prialnik et al., 2004). Thus OCCs are an opportunity to directly sample relatively pristine remnants of the earliest 
era in the Solar System. Within the broad category of OCCs, there is also an important subclass called Manx (tail-less) comets. While most OCCs are thought to be icy in nature, some Manx comets may be composed of more silicate rich material, potentially similar to S-type asteroids. Thus some Manx comets may represent a population of scattered inner solar system objects (Meech et al., 2016). Exploration of a Manx comet would thus test hypotheses about early planetesimal composition and dynamic models of giant planet migration.

\subsection{Interstellar Objects}

ISO's are thought to be planetesimal remnants that have been ejected out of their own solar system (Raymond et al., 2018). While the existence of these objects was theorized for decades, it has only recently been confirmed by the detections of the interstellar objects 1I/'Oumuamua in 2017 and 2I/Borisov in 2019. The two known ISOs differ significantly, however. Observations of 1I/'Oumuamua revealed an elongated object with a radius of $\sim 100 \mathrm{~m}$, an axial ratio of 5-10:1, and no visible coma (Meech et al., 2017). In contrast, 2I/Borisov is an active comet with a nucleus several $\mathrm{km}$ in radius (Jewitt and Luu, 2019). Compositionally, 2I/Borisov matches the prediction that most ISOs should be icy bodies (Engelhardt et al., 2017) although depleted in carbon-chain species (Fitzsimmons et al., 2019; Opitom et al., 2019). In contrast, the composition of 1I/'Oumuamua remains uncertain; spectroscopic observations suggest its reddish color could be either cometary or asteroidal in origin (Meech et al., 2017). Overall, ISOs represent an unique opportunity to test the diversity of planetesimals among exoplanetary systems.

Science Question Summary - In order to use ISOs and OCCs to understand fundamental questions about the solar system formation, detailed information on refractory and volatile and isotopic composition, as well as on structure and density are needed.

\section{Limitations of Telescopic Observations of ISOs/OCCs}

Recent improvements in observational capabilities have significantly enhanced the detection rates of ISOs and OCCs. Since 2010, Pan-STARRS (Kaiser et al., 2002) has committed to performing a systematic survey of the entire Hawaiian sky. Pan-STARRS performed the first confirmed detection of an ISO as it passed through our solar system: 1I/'Oumuamua (Meech et al., 2017). At an apparent magnitude of 22.5, this object would have been undetectable with previous sky surveys. Pan-STARRS also allowed for the advanced detection of OCCs such as comets C/2011 L4 (PANSTARRS) and C/2017 K2 (PANSTARRS) discovered two and five years before their perihelia respectively. Beyond Pan-STARRS, the upcoming Vera C. Rubin Observatory (LSST) will be able to detect even fainter objects, and has committed $90 \%$ of its telescope time over the first ten years to performing a sky survey. This will increase predicted ISO detection rates up to 1/year (Trilling et al., 2017). With first light scheduled for 2020/2021, these new facilities will strongly influence our catalog of solar system objects over the next decade.

Actively outgassing OCCs are some of the brightest known comets. Spacecraft observation of an OCC or ISO would reveal the surface topography and outgassing behavior with an order of magnitude higher resolution than telescopic observations. Furthermore, in-situ spectroscopy of dust or outgassed materials could reveal key chemical markers that can shed light on the object accretional environment. 
Observing tail-less objects like 1I/'Oumuamua or Manx comets is even more challenging. Despite a global telescopic observational campaign of 1I/'Oumuamua, many basic questions remain unresolved. The ISO's reddish color could be consistent with either an asteroidal or cometary origin (Meech et al., 2017; Fitzsimmons et al., 2018), and while non-gravitational cometary-like acceleration was detected (Micheli et al., 2018), no direct spectroscopic evidence of outgassing was found (Trilling et al., 2018). Retiring these ambiguities requires a dedicated mission with high-resolution spectral and spatial imaging capabilities.
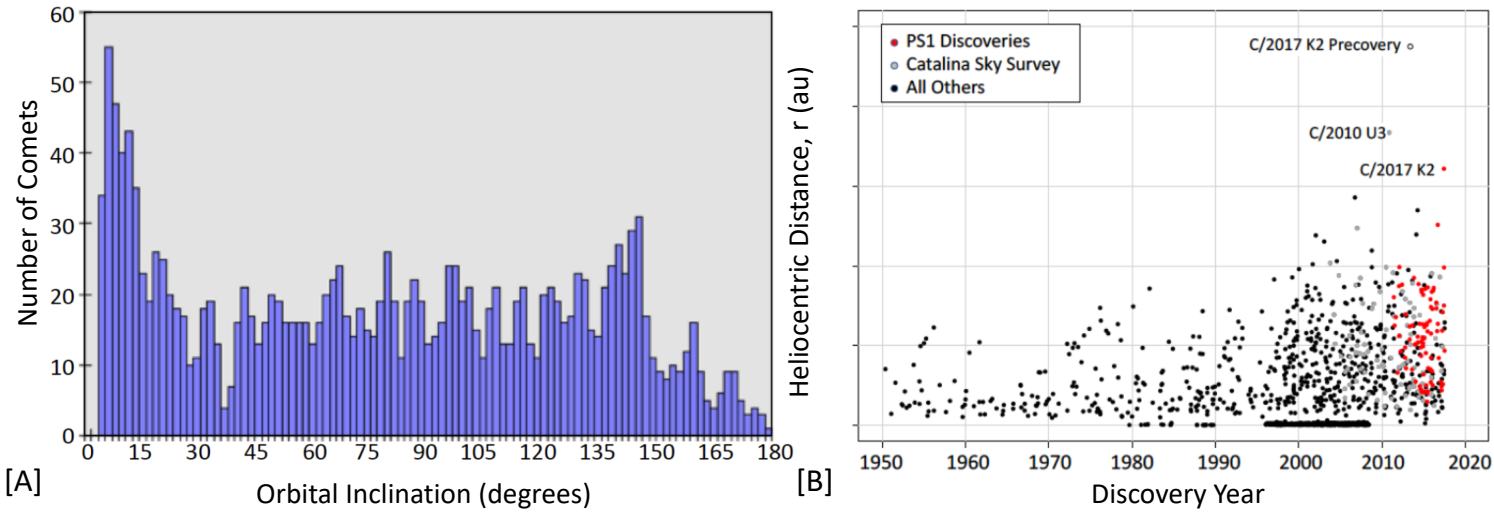

Figure 2: [A] Distribution of Oort Cloud Comet orbital inclinations Willman Jr (1995). [B] Orbital trajectory of 1I/'Oumuamua through the solar system (Meech et al. 2017).

We advocate for continued support to make telescopic resources available to the search for and global characterization of ISOs and OCCs in order to prepare for dedicated spacecraft missions that will answer detailed ISO/OCC science questions.

\section{Implementation: Reactive Rapid Response Missions}

ISOs and OCCs have unique orbital properties that strongly constrain mission design. Unlike other comets or asteroids, ISOs and OCCs may approach the solar system from any angle, including perpendicular to the ecliptic (see Fig. 2). For an arbitrary ISO or OCC, the geometry of Jupiter is unlikely to be statistically favorable for a gravity assist to leave the ecliptic. Thus the most favorable mission architecture is to encounter the ISO or OCC at the ecliptic, near perihelion in the inner solar system. Even with longer discovery lead times ( $\sim 5$ years $)$ with new surveys, this requires a response more rapid than allowed by the current paradigm. However, such a mission requires a rapid-response architecture in order to account for the short discovery window of ISOs and OCCs. ISOs in particular are not bound to the Sun and so will follow a hyperbolic trajectory through space with much faster velocities than standard solar system objects: 1I/'Oumuamua and 2I/Borisov reached hyperbolic excess velocities, $\mathrm{v}_{\infty}=26.2 \mathrm{~km} / \mathrm{s}$ (Meech et al., 2017) and $32.2 \mathrm{~km} / \mathrm{s}$ (Bailer-Jones et al., 2020) relative to the Sun. Below, we describe completed mission concept studies demonstrating the viability of missions to OCCs and ISOs using rapid response strategies.

\subsection{SmallSat missions}

Xenia is a 2019 NASA-JPL mission concept (Donitz et al., 2020) to explore the OCC 2017/K2, which reaches perihelion at 2.3 au in 2022. This first-of-its-kind concept would use twin SmallSat spacecraft to explore comet diversity and constrain the temperature at the edge of the protoplanetary disk via a ultraviolet spectrometer and narrow-angle camera. Xenia is nominally designed 
for a hypothetical encounter with $\mathrm{C} / 2017 \mathrm{~K} 2$ in 2022, but the concept's projected rapid turnaround time (18-24 months from start to launch) makes it an ideal blueprint for smallsat reactive missions to other future OCCs or ISOs in the future. However, there is no programmatic path for a mission concept like Xenia. Even with the smallsat architecture, the cost of the flight system does not fit within the cost cap (\$55M) of the Small Innovative Missions for Planetary Exploration (SIMPLEx) program. The extremely high launch energies required to meet the comet at perihelion requires a dedicated launch vehicle which currently falls outside of the scope of SIMPLEx . Actualizing a concept like Xenia within SIMPLEx would require a larger cost cap, an allowance for a dedicated launch vehicle, and a flexible launch window to respond to an OCC or ISO shortly after detection.

\subsection{Larger missions}

The 2019 NASA Planetary Science Summer Seminar (PSSS) team studied a flyby reconnaissance mission to a future ISO featuring an impactor (Moore et al., in review). The main spacecraft would use a camera to image geologic features, and near- and mid-IR spectrometers to identify prebiotic molecules, minerals, and ices. An impactor would excavate the ISO's interior, since the surface and interior compositions may differ through space weathering or internal differentiation. The impactor's guided navigation strategy would utilize a secondary camera, which would provide additional images up to $10 \mathrm{~m} /$ pixel. Finally, a UV spectrometer would image the impact plasma flash to quantify the elemental abundances of noble gases, key elements (C, H, O, N, P \& S), and isotopic ratios (e.g. $\delta^{16} \mathrm{O} / \delta^{18} \mathrm{O}$ ). This flash would also increase the signal to noise ratio for other instruments on board the main spacecraft and ground-based observations. The spacecraft would be kept in ground storage for up to seven years until detection of a suitable ISO. While designed for a hypothetical New Frontiers Announcement of Opportunity, the concept has significant cost reserves (46\% for Phases A-D) and could be adapted to fit a Discovery cost cap.

ISOs/OCCs are viable targets for SmallSat, Discovery, and New Frontiers missions using a rapid response strategy. A separate white paper was submitted to the Decadal Committee by Donitz, et. al for the August 15th deadline endorsing a New Frontiers mission concept study to encounter an Oort Cloud Comet.

\section{Enabling Technologies and Policies}

Sending a spacecraft to an ISO or OCC is a unique technical and programmatic challenge. The objects have high velocities, unusual orbital trajectories, and are only discoverable a few years at best before their perihelion. Missions to OCCs and ISOs therefore require a flexible, rapid response-style architecture (Donitz et al. 2020). However, logistical and programmatic barriers within NASA currently prohibit rapid response strategies in mission proposals. Here we outline the required changes to current policies to enable a reactive mission to an ISO or OCC.

Allow for rapid launch turnaround times. Rapid response missions must launch quickly in response to discovery of a compelling target. For example, Moore et al. found that NASA must launch a spacecraft within 3-6 months of target detection to successfully encounter an ISO, even assuming a high-performance launch vehicle. This estimate is based on the small number of known ISOs, faster turnaround times allow a greater percentage of incoming targets to be reached. 
We recommend the Decadal Committee examine changes to the current mission announcements of opportunities in order to increase launch flexibility for rapid (months or less) launch vehicle acquisition turnaround times.

\subsection{Discovery and New Frontiers Recommendations}

NASA's Discovery and New Frontiers programs currently require long waiting periods between a mission's initial proposal and eventual launch. For example, the Discovery 2019 AO was released in April 2019, to select two missions with launch dates between 2025-2029 (NASA, 2019a). This presents a significant barrier to exploring targets such as ISOs and OCCs, which can only be discovered a few years (at best) before perihelion and quickly escape the solar system. However, this challenge can be overcome by making specific changes to the Discovery and New Frontiers AOs in order to allow rapid-response style missions.

1. Allow for flexible mission targets. Proposals must be allowed to wait to choose a specific target (from a general class) until after submission, as fast targets such as OCCs or ISOs may not be discovered until shortly before (or even after) their perihelion.

2. (Discovery only) Allow proposals to include planned long-term spacecraft storage, either in a space-based parking orbit or on the ground, until a suitable target is discovered.

\subsection{Smallsat/SIMPLEx recommendations}

The SIMPLEx program is one of NASA's primary proposal pipelines for smallsat missions, which are primarily proposed as ride-along missions of opportunity (secondary payloads that hitch a ride with larger missions) (NASA, 2019b). As Xenia demonstrated and as discussed earlier in this paper, the low cost cap, ride-share requirement, and rigid AO structure and launch window prohibit the exploration of an OCC or ISO within the current SIMPLEx paradigm. An ISO or OCC SmallSat mission requires a more flexible programmatic path than the current SIMPLEx paradigm. We recommend that NASA revisit the funding mechanisms for smallsat missions to enable reactive missions:

1. Increase the SIMPLEx cost cap for SmallSat spacecraft missions

2. Solicit NASA SIMPLEx proposals annually in order to facilitate reactive missions.

3. Add mechanisms to unlock resources to start projects within months of object discovery to meet strict launch periods, reduce time to acquire long-lead items, and allow for shorter development schedules.

4. Allow SIMPLEx missions to use dedicated launch vehicles. It is unlikely that a primary spacecraft would be headed in the correct direction at the correct time to host a ride-along mission to a newly discovered extraordinary target. Dedicated launch vehicles would leverage the advantages SmallSats as fast, responsive spacecraft (small mass, high $\Delta v$ ), and enable rapid response missions to a wider variety of targets.

5. Minimize cost penalties for standalone launch vehicles. Any cost penalty imposed for using a standalone launch vehicle should be significantly smaller than the original launch vehicle purchase price. 


\section{Interdisciplinary Synergy}

Implementing our proposed programmatic and policy changes to enable rapid response mission architectures would achieve interdisciplinary science across multiple NASA Divisions-a key goal for the coming decade (Mandt, K. E. and et al., 2019).

Planetary Defense - Reports published by NASA's Small Bodies Assessment Group (Mainzer, Amy and et al., 2020) and the US Executive Branch's National Science and Technology Council, Interagency Working Group for Detecting and Mitigating the Impact of Earth-Bound Near-Earth Objects (2018) both advocate for the ability to rapidly deploy spacecraft to explore and defend against Near-Earth Objects. Thus, programmatic changes to allow for rapid response missions to ISOs and OCCs will also meet critical needs within the planetary defense community.

Astrophysics - Direct observations of ISOs represent an opportunity for cross divisional science with the NASA Astrophysics Division(National Research Council, 2010), since science done at interstellar objects are direct samples of exoplanetary systems.

\section{Summary}

Oort cloud comets and Interstellar objects hold key information regarding the early history of our solar system and the nature of exoplanetary systems. Telescopic capabilities are now sufficiently advanced to regularly discover these objects (as evidenced by the detections of ISOs 1I/2017 'Oumuamua and 2I/2019 Borisov), but understanding their full complexity will require a dedicated spacecraft mission. Multiple teams have demonstrated that Discovery, New Frontiers, and SmallSat/SIMPLEX missions to these objects are feasible and would return high-value science. However, reaching these objects requires a reactive mission architecture which is incompatible with current NASA planetary science mission proposal policies that require a specific target to be named when the proposal is submitted. We advocate for specific revisions to NASA policies to enable reactive mission architectures to explore these unique, once-in-a-lifetime visitors.

\section{References}

Bailer-Jones, C. A. L., Farnocchia, D., Ye, Q., Meech, K. J., Micheli, M., 2020. A search for the origin of the interstellar comet 2I/Borisov. A\&A 634, A14.

Dones, L., Brasser, R., Kaib, N., Rickman, H., 2015. Origin and Evolution of the Cometary Reservoirs. Space Sci. Rev. 197 (1-4), 191-269.

Donitz, B., Matousek, S., Castillo-Rogez, J., et al, 2020. Smallsat reactive flyby to oort cloud comets and interstellar objects. In: Interplanetary Small Satellite Conf., \& Pasadena, CA.

Engelhardt, T., Jedicke, R., Vereš, P., et al, 2017. An Observational Upper Limit on the Interstellar Number Density of Asteroids and Comets. AJ 153 (3), 133.

Fitzsimmons, A., Hainaut, O., Meech, K. J., et al, 2019. Detection of CN Gas in Interstellar Object 2I/Borisov. ApJ 885 (1), L9.

Fitzsimmons, A., Snodgrass, C., Rozitis, B., et al, 2018. Spectroscopy and thermal modelling of the first interstellar object 1I/2017 U1 'Oumuamua. Nature Astronomy 2, 133-137.

Jewitt, D., Luu, J., 2019. Initial Characterization of Interstellar Comet 2I/2019 Q4 (Borisov). ApJ 886 (2), L29. 
Community White Paper to the Planetary Science \& Astrobiology Decadal Survey (2023-2032)

Kaiser, N., Aussel, H., Burke, B. E., et al, 2002. Pan-STARRS: A Large Synoptic Survey Telescope Array. In: Tyson, J. A., Wolff, S. (Eds.), procspie, Volume 4836 of Society of Photo-Optical Instrumentation Engineers (SPIE) Conference Series, pp. 154-164.

Mainzer, Amy, et al., 2020. The Future of Planetary Defense in the Era of Advanced Surveys: A White Paper Commissioned by SBAG for the 2023-2032 Plan. Sci. \& Astrobio. Decadal Survey.

Mandt, K. E., et al., 2019. Advancing Space Science Requires NASA Support for Coordination Between the Science Mission Directorate Communities. White Paper submitted to the Astrophysics, Heliophysics, and Planetary Science Decadal Surveys.

Meech, K. J., Weryk, R., Micheli, M., et al, 2017. A brief visit from a red and extremely elongated interstellar asteroid. Nature 552 (7685), 378-381.

Meech, K. J., Yang, B., Kleyna, J., et al, 2016. Inner solar system material discovered in the Oort cloud. Science Advances 2, e1600038.

Micheli, M., Farnocchia, D., Meech, K. J., et al, 2018. Non-gravitational acceleration in the trajectory of 1I/2017 U1 ('Oumuamua). Nature 559, 223-226.

Moore, K. M., Courville, S. W., Ferguson, S., et al. Bridge to the stars: A mission concept to an interstellar object.

NASA, 2019a. Announcement of Opportunity: Discovery 2019.

NASA, 2019b. Announcement of Opportunity: NASA's Third Stand Alone Missions of Opportunity Notice (SALMON-3).

National Research Council, 2010. New Worlds, New Horizons in Astronomy and Astrophysics. The National Academies Press, Washington, DC.

National Science and Technology Council, Interagency Working Group for Detecting and Mitigating the Impact of Earth-Bound Near-Earth Objects, 2018. National Near-Earth Object Preparedness Strategy \& Action Plan. Exec. Office of the President of the United States, WashDC.

Opitom, C., Fitzsimmons, A., Jehin, E., et al, 2019. 2I/Borisov: A $\mathrm{C}_{2}$-depleted interstellar comet. $A \& A$ 631, L8.

Prialnik, D., Benkhoff, J., Podolak, M., 2004. Modeling the structure and activity of comet nuclei, pp. 359.

Raymond, S. N., Armitage, P. J., Veras, D., 2018. Interstellar Object 'Oumuamua as an Extinct Fragment of an Ejected Cometary Planetesimal. ApJ 856 (1), L7.

Raymond, S. N., Izidoro, A., 2017. Origin of water in the inner Solar System: Planetesimals scattered inward during Jupiter and Saturn's rapid gas accretion. Icarus 297, 134-148.

Trilling, D. E., Mommert, M., Hora, J. L., et al, 2018. Spitzer Observations of Interstellar Object 1I/‘Oumuamua. AJ 156 (6), 261.

Trilling, D. E., Robinson, T., Roegge, A., et al, 2017. Implications for Planetary System Formation from Interstellar Object 1I/2017 U1 ('Oumuamua). ApJ 850 (2), L38.

Willman Jr, A. J., 1995. Implications of Magnitude Distribution Comparisons between TNOs and Comets. http://www.princeton.edu/ willman/tno-comet/tnocomet.pdf. 